\title{
Application of hollow pollen grains as scaffolding building blocks for post-surgical bone cancer treatment
}

\author{
Solmaz Zakhireh ${ }^{1}$, Yadollah Omidi², Younes Beygi-Khosrowshahi ${ }^{3}$, Elham Mohajel \\ Kazemi $^{4}$, Jaleh Barar ${ }^{2}$, and Khosro Adibkia ${ }^{2}$ \\ ${ }^{1}$ Tabriz University of Medical Sciences \\ ${ }^{2}$ Tabriz University of Medical Sciences Faculty of Pharmacy \\ ${ }^{3}$ Azarbaijan Shahid Madani University Faculty of Engineering \\ ${ }^{4}$ University of Tabriz
}

April 28, 2020

\begin{abstract}
After surgical removal of bone tumors, elimination of the remains of cancer cells along with tissue healing and functionality is a therapeutic goal. Regarding the destructive effect of chemo-/radiotherapy on healthy cells, the development of multimodal scaffolds with simultaneous anticancer and osteo-regenerative potency is of particular importance as regenerative medicine for bone tissue engineering. Our previous study demonstrated that hollow pollen grain (HPG) of Pistacia vera L. offers a unique bone-forming activity and encapsulating capacity that it can be considered as an excellent scaffolding building block in bottomup bone engineering. In the present study, for the first time, the anticancer potential of Pistacia vera L. HPG was investigated in-vitro using human osteosarcoma cell line MG63. Optical imaging of the HPG-cell interface indicated large focal adhesion due to the HPG unique surface features such as functionality and ornamentation. MTT assay results showed an anti-proliferative effect of HPG on the MG63 cells. Moreover, from the gene expression and DAPI staining analyses, HPG of Pistacia vera L. was found to be an apoptosis-inducing building block against MG63 bone cancer cells. So therefore, such a multifunctional building block with the ability of discriminatory killing human osteosarcoma cell line is proposed to be used after surgery to not only hinder cancer recurrence after surgery but also to stimulate bone healing.
\end{abstract}

\section{Hosted file}

Pollen anticancer scaffolding building block.docx available at https://authorea.com/users/310312/ articles/441258-application-of-hollow-pollen-grains-as-scaffolding-building-blocks-for-postsurgical-bone-cancer-treatment 